\title{
Apparent motion during saccadic suppression periods
}

\author{
Robert Scott Allison · Jens Schumacher • \\ Shabnam Sadr $\cdot$ Rainer Herpers
}

Received: 26 February 2009 / Accepted: 30 November 2009 / Published online: 19 December 2009

(C) Springer-Verlag 2009

\begin{abstract}
Sensitivity to many visual stimuli, and, in particular, image displacement, is reduced during a change in fixation (saccade) compared to when the eye is still. In these experiments, we studied the sensitivity of observers to ecologically relevant image translations of large, complex, real world scenes either during horizontal saccades or during fixation. In the first experiment, we found that such displacements were much less detectable during saccades than during fixation. Qualitatively, even when trans-saccadic scene changes were detectible, they were less salient and appeared slower than equivalent changes in the absence of a saccade. Two further experiments followed up on this observation and estimated the perceived magnitude of trans-saccadic apparent motion using a two-interval forcedchoice procedure (Experiment 2) and a magnitude estimation procedure (Experiment 3). Both experiments suggest that trans-saccadic displacements were perceived as smaller than equivalent inter-saccadic displacements. We conclude that during saccades, the magnitude of the apparent motion signal is attenuated as well as its detectability.
\end{abstract}

Keywords Saccadic suppression - Displacement . Motion $\cdot$ Visual perception $\cdot$ Saccades

R. S. Allison $(\bowtie) \cdot$ J. Schumacher $\cdot$ S. Sadr Department of Computer Science and Engineering, Centre for Vision Research, York University, 4700 Keele St., Toronto, ON M3J 1P3, Canada e-mail: allison@cse.yorku.ca

J. Schumacher $\cdot$ R. Herpers

Department of Computer Science,

Bonn-Rhein-Sieg University of Applied Sciences,

Sankt Augustin, Germany

\section{Introduction}

A distinguishing feature of the human eye is that highest acuity is restricted to small portion of the central retina called the fovea. To appreciate a natural or computer-generated scene, fast eye movements called saccades must direct this foveal region to areas of interest in the scene. During these saccades, images of objects stream across the retina at hundreds of degrees per second. Despite this disjoint motion on the retina, the world does not normally appear disjoint or unstable and motion blur during saccades is not apparent. Part of the reason is that, during saccades, sensitivity to visual stimuli is reduced-an effect known as saccadic suppression (Dodge 1903; Holt 1903; Volkmann et al. 1978; Burr et al. 1982).

Saccadic suppression has been the subject of much controversy. The phenomenon itself is robust and there is no debate that some stimuli are more difficult to see during a saccade. However, the degree, if any, that the visual system actively suppresses visual responses during a saccade is more controversial. Some trans-saccadic loss of sensitivity occurs in the retinal image itself. Finite visual integration times and the rapid motion of the stimulus results in motion blur and reduced illumination of the photoreceptor array (Matin 1974). Thus, high spatial-frequency information in the stimulus will be reduced in contrast during a saccadic eye movement, perhaps below visibility. Experiments with simulated saccadic image motions displayed during fixation though have demonstrated that some stimuli are not apparent during saccades that are visible during simulated saccades suggesting active suppression (Diamond et al. 2000). Conversely, some stimuli can be seen better during saccades if the eye movement reduces the instantaneous retinal velocity or temporal frequency of the target (Castet and Masson 2000). 
Many have argued that a process of active suppression is normally unnecessary. In viewing moderate to high-contrast visual scenes, the low-contrast motion-blurred transsaccadic stimulus is preceded and followed by more salient images during the fixation period before and after the saccade. Since the early days of studying the phenomenon, some investigators have proposed that the trans-saccadic stimulus is masked by these more salient stimuli (Dodge 1905). In modern times, this proposal has been prominently put forward by MacKay (1970) and Campbell and Wurtz (1978). Indeed, some have argued (Wurtz 2008) that forward and backward masking phenomenon may have evolved specifically to serve this ecological role. Such masking cannot explain the suppression of isolated stimuli presented in otherwise dark environments that has been reported in psychophysical and electrophysiological studies. Additional support for active suppression has come from imaging and electrophysiological studies claiming reduction in visual responses to isolated stimuli around the time of saccades (Thiele et al. 2002; Kleiser et al. 2004; Thilo et al. 2004). The reductions occur when the stimulus is presented just prior and post-saccade eliminating explanations based on image quality during the saccade. Typically the magnitude of the firing rate response of a cell exhibiting suppression is reduced when the stimulus is presented peri-saccadically rather than during steady fixation (Thiele et al. 2002). However, this active suppression of visibility is rather modest and it is possible that masking phenomena are responsible for most saccadic suppression in natural, well-lit environments (Wurtz 2008).

Interestingly, if the retinal image motion normally presented during saccades is compensated for by imposed image motion to retinally stabilize the display, then subjects can see the high frequency stabilized images that would normally be subject to motion blur (Castet and Masson 2000; Garcia-Perez 2001). In fact, saccadic suppression seems to be most pronounced at lower spatial frequencies that would be spared contrast reduction due to motion blur during the saccade (Burr et al. 1994; Ross et al. 1996). This selective suppression of low spatial frequency patterns seems to reflect a more general selective saccadic suppression of the magnocellular pathway as argued by Burr et al. (1982, 1994, 1999) (Ross et al. 1996).

Besides the spatial frequency selectivity, there is evidence that motion mechanisms are selectively suppressed, that suppression depends on the chromatic content of stimulus and specifically that saccadic suppression of displacement is not apparent for isoluminent displays (Burr et al. 1994; Bridgeman and Macknik 1995; Uchikawa and Sato 1995; Anand and Bridgeman 2002)_characteristics that lend support to the proposal of a selective suppression of magnocellular rather than parvocellular pathways. If suppression of magnocellular motion sensitive mechanisms occurs, then the trans-saccadic apparent motion signal may be suppressed which would minimize the disruption from trans-saccadic motion/displacement of the retinal image. However, to detect an actual displacement or movement of objects in the visual world during a saccade, observers would need to rely on comparison of pre- and post-saccadic position to detect trans-saccadic apparent motion.

Burr et al. (1982) found that subjects' sensitivity to transient changes in the velocity of a drifting grating was much lower trans-saccadically than during fixation. Furthermore, although supra-threshold transitions clearly resulted in disturbed motion perception during fixation, only "a vague sense that an untoward event occurred" was reported transsaccadically. Ilg and Hoffmann (1993) measured sensitivity to movement of a large repetitive background image. In one set of experiments, a large projected grid pattern was moved by means of a galvanometer in the optical path of the projector. Participants judged whether the background moved when a sweep of the background was presented at various times relative to the onset of a saccade between two targets. The amplitude of the $20 \mathrm{~ms}$ galvanometer sweep was adjusted to threshold in a staircase procedure. Thresholds were elevated trans-saccadically so that during a $20^{\circ}$ saccade the background needed to move by $5.4^{\circ}$ over $20 \mathrm{~ms}$ to be apparent (average velocity of $270 \%$ s) while during fixation the threshold could not be measured due to device limits but was at least 32 times smaller. All subjects reported that trans-saccadic sweeps appeared to occur after the saccade and were "slow and sluggish". In the present paper, we follow up on these earlier anecdotal reports by measuring the apparent magnitude of supra-threshold transsaccadic apparent motion.

Saccadic suppression of motion or displacement is thus consistent with increased saccadic suppression in the magnocellular system. However, additional considerations suggest that the saccadic suppression of displacements involves other mechanisms as well. During a saccade, the visual world moves across the retina but this does not necessarily imply anything moved in the world. In order to estimate the physical motion of objects in the scene, the retinal motion due to the eye movement needs to be taken into account. This process could involve retinal information (i.e. retinal flow) or extra-retinal information, such as efference copy, or eye muscle proprioception with neurophysiology suggesting a major role for corollary discharge (CD) (for review, see Wurtz 2008). Thus, detection of displacement relies not only on detection of change in retinal position but also on estimation and compensation for a large, variable and noisy eye motion signal.

Though saccadic suppression has evolved in the context of trans-saccadic motion of the visual world during gaze movements, it has not typically been studied using ecologically relevant motion of the entire visual scene. Retinal 
motion of the entire visual field is the typical stimulus during saccades. How does saccadic suppression generalize to these ecological conditions? The most relevant study is McConkie and Currie (1996), who conducted a study to determine whether saccadic suppression generalized to more naturalistic stimuli and image transformations for stimuli presented on a small computer screen. It was found that detection rate was a function of saccade duration, and as saccade duration increased, probability of detection dropped. In the present experiments, we extended these findings by studying the sensitivity of observers to transsaccadic image translations of large, complex real world scenes presented using a CRT projector. We also asked whether the perceived magnitude of the displacement of the visual field is affected during saccades or just its visibility.

\section{General methods}

System setup

A real-time video eye-tracking system (Vision 2000, EL-MAR Inc., Toronto, Canada) based on adaptive real-time image processing was used to obtain accurate measurements of the eye movement. The eye position was measured at a frequency of $120 \mathrm{~Hz}$ and sent to the workstation over a RS232 serial port at a baud-rate of 38400 . A standard Linux workstation (dual AMD Athlon MP 1900 + processors, 1 GB System RAM, GeForce4 TI4600, RedHat Linux with kernel version 2.4.18) was used to display the stimuli, process eye-movement data and perform the changes in the image for the experiments. For the final experiment the image generation hardware was upgraded (Intel ${ }^{\circledR} \mathrm{Xeon}^{\circledR}$ at $3.2 \mathrm{GHz}$ Dual CPU, 4 GB System RAM, GeForce PCX5750 graphics adapter) but image generation and display specifications were maintained.

The images were projected onto a rear-projection screen $\left(2.4 \mathrm{~m}^{2}\right.$, High Contrast 'Blackscreen', Draper Inc, Spiceland, IN) via a Barco 808 CRT projector with a resolution of $1,024 \times 768 \times 120 \mathrm{~Hz}$. The full projector image subtended $64^{\circ}$ by $48^{\circ}$ at the viewing distance of $80 \mathrm{~cm}$. Subjects sat at a table with head supported in a chin cup. Viewing was binocular. The experiment was performed in a dark laboratory and the black level of the projector was adjusted so that the edge of the video frame was not apparent.

\section{Subjects}

Subjects with normal ocular motility and vision were recruited. Six subjects served in experiment 1 ( 1 female, 5 male, ages 22-38). Three subjects were familiar with the eye-tracking system. The other three subjects were completely naïve to the entire procedure. In experiment 2 , four naïve and one experienced subject participated ( 1 female, 4 male, ages 22-39). In experiment 3 , data were collected from one experienced and nine naïve subjects ( 6 females, 4 males, ages between 22 and 35).

The study was approved by research ethics committee at York University and performed in accordance with the ethical standards laid down in the 1964 Declaration of Helsinki. All persons gave their informed consent prior to their inclusion in the study.

\section{Material}

Images of complex natural scenes were recorded with a digital camera (5.1 megapixel Nikon Coolpix5400) and down-sampled to $1,056 \times 792$ pixels. The images were larger than the display so that image translations could be performed without shifting the border. Sample images are shown in Fig. 1.

\section{General procedure}

Participants viewed images in a random order and were told to analyse and memorize the scenes for a later memory task. Eye position data were continuously recorded and monitored in real time. During the trials, saccade-contingent displacements of the image were imposed. The displacements were vertical or horizontal image shifts of the entire image on the display.

Translations were randomly to the right or to the left (also up and down in Experiment 3), triggered at the second saccade (third in Experiment 1) of sufficient duration that was detected in the viewing period. Given that subjects were free viewing the stimulus, this meant that the changes occurred at unpredictable intervals for the subjects. For each trial, the saccade-contingent change was specified to be either trans-saccadic, inter-saccadic or a no-change catch trial (Fig. 2). Trans-saccadic changes occurred immediately after detecting the saccade (see "System end-to-end latency" for timing). Inter-saccadic changes occurred after a random time interval from the start of the trial (Experiment 1) or $150 \mathrm{~ms}$ after the saccade trigger (Experiments 2 and 3). Programming the inter-saccadic change to occur following a saccade led to greater reliability in placing the change during a fixation. In some trials no change was specified and the image was not displaced.

\section{Saccade detection and prediction}

We required real-time triggering of saccade-contingent changes during naturalistic scene viewing. Since the size, direction and timing of saccades were not predictable, simple velocity thresholding or other schemes could not be 

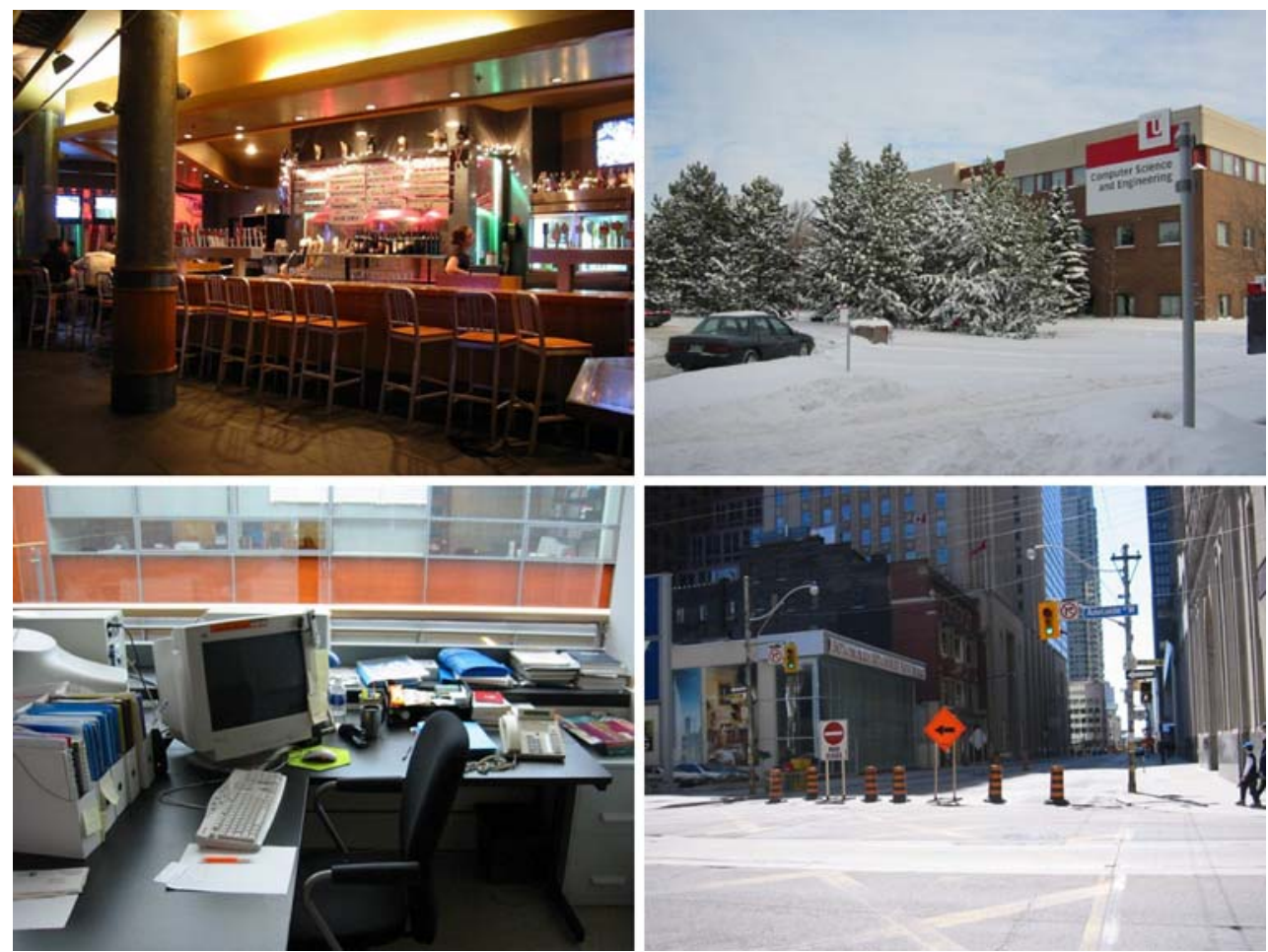

Fig. 1 Sample images shown to the participants in the three experiments. These four images were part of a set of eighty images of natural and constructed environments

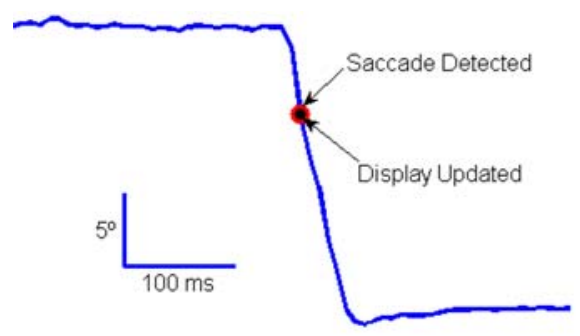

Fig. 2 Timing of saccade-contingent image displacements. Eye movement is shown as a function of time (proceeding to the right). The left--hand panel shows the timing of the scene change compared to the trigger for a trans-saccadic displacement. The change is programmed to occur as soon as the software detects the trigger condition (see text

used. The algorithm used and its evaluation is discussed in "Appendix". Saccade detection was based on the horizontal component of the eye movement. A saccade duration of $58 \mathrm{~ms}$ was set as a lower bound limit for making a transsaccadic change. To ensure that the transition occurred during the saccade, an algorithm was needed to detect a saccade and predict its duration based on the initial portion of the saccade (i.e. the first two samples). A five-point differentiator was used to estimate current velocity. A saccade was indicated if the filtered velocity exceeded a threshold of $60 \%$ and the eye was accelerating for the previous two frames. On average, saccades that met this threshold criterion occurred every $1.7 \mathrm{~s}$.

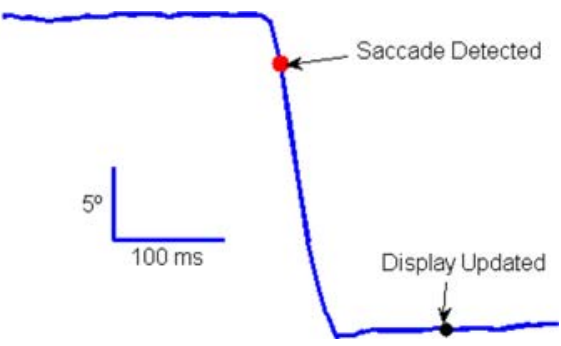

for details on system delay). The right-hand panel shows an inter-saccadic displacement where the change is programmed to occur $150 \mathrm{~ms}$ after the trigger-assumed to be during fixation due to the refractory period for saccades

\section{System end-to-end latency}

The time between the saccade onset and the actual display change was measured. A photodiode detector attached to the screen indicated changes of the display luminance. The horizontal eye position, provided by the analogue output of the eye-tracker, indicated the progression of a saccade. A large, full-field luminance change in the display was triggered by the saccade crossing a known threshold. The endto-end latency between the saccade crossing this threshold and the display luminance change could be monitored with a Kikusui 5020A oscilloscope with sufficient precision. The time-base used was $20 \mathrm{~ms} / \mathrm{div}$ ( $2 \mathrm{~ms}$ under magnification) 
with vertical sensitivity of $5 \mathrm{~V} / \mathrm{div}$ on the eye-tracker position signal and $1 \mathrm{~V} /$ div on the photo diode signal. Requiring the subject to switch fixation between two targets separated horizontally by $20^{\circ}$ controlled the amplitude of the saccades executed during this test. The average duration of such a movement was $67 \mathrm{~ms}$. The average delay from eye position threshold to graphic update was $35 \mathrm{~ms}$. This includes delays related to serial data stream processing, numerical differentiation and saccade prediction, rendering, graphics update and display. These measurements do not include the internal delay of the eye-tracker which is uniformly distributed between 8 and $16 \mathrm{~ms}$ depending at what point in the video frame the saccade started. Hence, we can estimate the total end-to-end latency as between 43 and $51 \mathrm{~ms}$.

\section{Calibration}

Each session started with a calibration procedure where the subject sequentially fixated ten points, five horizontal and five vertical. The horizontal points were evenly distributed between $-15^{\circ}$ and $+15^{\circ}$ and the vertical points between $-12^{\circ}$ and $+12^{\circ}$ of viewing angle. If the internal routine of the eye tracker indicated that the calibration was successful, we started the trial. The quality of the calibration was also manually checked on a plot of the fit of the calibration points to the estimated data and the accuracy of gaze contingent display of a fixation-slaved marker overlaid on the calibration screen.

\section{Experiment 1: detectability of trans-saccadic image translation}

In the first experiment, the sensitivity of subjects to translation of complex real world images during a saccade was investigated and compared with sensitivity to translation in the absence of a saccade. The subjects freely viewed a complex scene, because we were interested in suppression during natural as opposed to prescribed gaze behaviour. After a specified number of saccades, the entire scene was translated horizontally either during the saccade or during a fixation.

We varied the size of the translations as there is some evidence from experiments with small target displays that displacements that are small with respect to the size of the saccade are less detectable ( $\mathrm{Li}$ and Matin 1990; McConkie and Currie 1996; MacAskill et al. 2003). ${ }^{1}$ It was interesting to investigate whether this was also true for translations of the entire visual environment.

\footnotetext{
${ }^{1}$ Note that Bridgeman et al. (1975) found similar effects of shift size for a larger $13^{\circ} \times 13^{\circ}$ array of elements.
}

Methods

Participants examined a set of 45 images, randomly ordered. On each trial, the image of the scene was displayed for $10 \mathrm{~s}$. After the image, a black screen cued the subjects to indicate whether there was an apparent displacement or not during the image presentation via a key press. Following the response, the next trial began. The frequency of actual changes was not communicated to the subjects. The change was a translation of the whole scene to the left or the right (with equal frequency) and each scene was changed once per trial (or not changed). An example of the type of change that would occur was shown to the subjects prior to the experiment.

Trials were divided as follows. Ten trials were assigned to each of three levels of saccade-contingent trans-saccadic change $\left(0.4^{\circ}, 0.8^{\circ}\right.$ and $\left.1.2^{\circ}\right)$. Ten trials were assigned to the no-change (catch trial) category. Five trials were assigned to timed (inter-saccadic) changes. These were $0.8^{\circ}$ horizontal translations and expected to be easily detected if the subjects did not coincidentally perform a blink or a saccade.

\section{Results and discussion}

It was found that saccades that met the detection criterion occurred at an average of 5.9 times per trial. Depending on the scene and subject, this number varied between 2 and 16 saccades. However, in some cases, the participant made insufficient large horizontal saccades to trigger the scheduled change. Also, our algorithm was conservatively designed and was able to reliably detect only large saccades but missed some long-duration saccades. In effect, these trials with scheduled changes that did not occur became additional catch trials. Out of 210 trials where a change was scheduled (30 displacements $\times 7$ subjects), data for 188 trans-saccadic displacement trials was obtained.

An analysis of false alarm and miss rates during the time-triggered (i.e. inter-saccadic) scene changes was performed. Responses could include two types of errors: reporting a change during the control condition where no change occurred (false alarm) and failing to detect when the image was changed (miss). Subjects acted conservatively with respect to change detection and made no false alarms during the no-change condition. In the timed-change (intersaccadic) condition, 5 out of 35 changes $(14.4 \%)$ were missed across the seven subjects. After analysis of the data, it was found that on four of these timed trials the change occurred during a coincidental saccade and one on a coincidental blink occurred. Thus, it appears that suppression during a coincidental saccade or blink explains why these usually obvious changes were missed.

In total, we obtained 188 trials with trans-saccadic scene changes. Over all the subjects, 86 of these changes were 
detected and 102 were not detected. The detection rate ranged from 32.3 to $67.6 \%$ in individual subjects. Only one subject in one session detected more changes than they missed. The average detection level was $44 \%$ and for most subjects was clearly less than $50 \%$.

The detection rate was higher than we expected based on previous reports from which detection probability could be inferred (Irwin 1992; MacAskill et al. 2003; Triesch et al. 2003). In this experiment, subjects were encouraged to view the scenes freely and to make many saccades. Although this permitted naturalistic eye movement behaviour, it did not allow for control of fixation location, duration or timing. However, the size and direction of saccades executed were known with respect to size and direction of transitions (recall that the scene changes in this experiment were always triggered on the horizontal component of saccades). To try to account for the higher than expected detection rate, detection rate was assessed as a function of saccade duration. This analysis indicated that it was possible that on some trials the saccade duration was too short to perform the change. The majority of changes were triggered with at least $33.2 \mathrm{~ms}$ remaining in the intra-saccadic interval. The total saccade duration in this category was typically about $50 \mathrm{~ms}$. Based on our total system delay measurements this is approximately equivalent to the time needed to finish a display change after the saccade started. The average time left after the detection of the saccade was $38 \mathrm{~ms}$ for the detected changes and $57.4 \mathrm{~ms}$ for undetected changes. This indicates that the duration of the saccade was a critical factor since suppression was more likely if the saccade duration exceeded the end-to-end latency. Thus, we conclude that most changes that were detected were the result of short saccade durations. Detection rate decreased if the saccade duration increased.

To eliminate detections based on short saccade duration in analysing the effects of translation magnitude, only saccades with duration of at least $66 \mathrm{~ms}$ were considered. For saccades with duration of at least $66 \mathrm{~ms}$, trans-saccadic changes were seldom noticed (Fig. 3). With a translation of $0.4^{\circ}$, only $6.2 \%$ of changes were detected, while $93.8 \%$ were not seen. Large translations were easier to see and the detection rate for $1.2^{\circ}$ translations was $17.6 \%$ while $82.4 \%$ of the changes were not detected. Thus, it was found that updates were typically not perceptible for saccades larger than $15^{\circ}$ or longer than $66 \mathrm{~ms}$ in duration.

This experiment demonstrates that trans-saccadic translational displacements are suppressed for stimuli filling a large part of the visual field, particularly for long duration saccades. This agrees with reports of saccadic suppression of displacement for simpler and/or smaller stimuli (Bridgeman et al. 1975; McConkie and Currie 1996). Interestingly subjects reported that the trans-saccadic changes were often not very salient and appeared 'slower' under

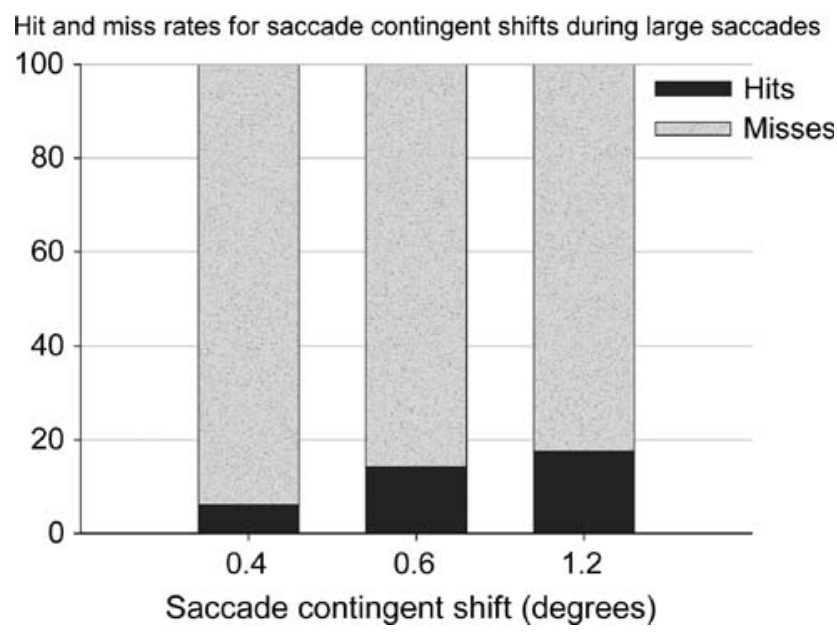

Fig. 3 Proportion of hits and misses for saccade-contingent translations during saccades longer than $66 \mathrm{~ms}$ duration (Experiment 1). Hit and miss rates are shown as a function of transition size

trans-saccadic conditions even when they were detected (in follow-up trials as they were not aware of which trials were trans-saccadic in the main experiment). In Experiments 2 and 3 , we follow up on this observation.

\section{Experiment 2: comparative magnitude of trans-saccadic versus inter-saccadic changes}

In Experiment 1, subjects were less sensitive to image displacements that occurred after a saccade onset but before the eye movement was completed (trans-saccadic) than to displacements that happened some time after a saccade took place and before another occurred (inter-saccadic). This was reflected in a low trans-saccadic detection rate and also in anecdotal reports that the trans-saccadic stimulus seemed slower than inter-saccadic stimuli. In this experiment, we attempted to measure this perceived slowing by having subjects match the perceived apparent displacement (typically accompanied by apparent motion) of a transsaccadic change with an inter-saccadic standard in a randomly ordered two-interval forced-choice (2IFC) procedure.

\section{Methods}

The same stimuli and apparatus were used as in Experiment 1 , although the psychophysical procedure differed. Five observers with normal vision and oculomotor motility participated.

Observers participated in 4-8 sessions. In each session, participants viewed 80 pairs of images in a randomly ordered 2IFC experiment. For each trial, in one randomly selected interval, there was a test stimulus displayed and in 
the other a comparison stimulus. The stimuli were presented for $2 \mathrm{~s}$ each and were separated by a $500 \mathrm{~ms}$ ISI when a black screen was displayed. The test could either be trans-saccadic or inter-saccadic. At the end of each trial, subjects responded (a) with a forced-choice judgement about which apparent displacement was larger (interval 1 or 2), (b) whether they detected a change in the first interval, and finally (c) whether they detected a change in the second interval. Subjects were provided with text prompts and responded with key presses.

Since presentation time was shortened (due to the large number of trials), changes were triggered after the second saccade in the stimulus interval (rather than the third as in experiment 1). One further change in the procedure was that the inter-saccadic changes were timed to occur $150 \mathrm{~ms}$ after the saccade occurred since it can be assumed that no further saccades can be performed in refractory period following the saccade. This procedure reduced the chance of a coincidental saccade occurring during or near the image translation. Data from each trial were still screened visually to ensure absence of artefact and to ensure that both the test and reference changes occurred as expected (inter-saccadic or trans-saccadic depending on condition).

The test stimuli were either $0.6^{\circ}$ or $1.2^{\circ}$ horizontal image translations and were either trans-saccadic or inter-saccadic. Thus, four psychometric curves were estimated for each test condition: $0.6^{\circ}$ trans-saccadic, $0.6^{\circ}$ inter-saccadic, $1.2^{\circ}$ trans-saccadic and $1.2^{\circ}$ inter-saccadic. The comparison stimulus displacements were randomly varied in discrete steps about the point of subjective equality based on pilot measurements $\left(0.075^{\circ}-0.75^{\circ}\right.$ for $0.6^{\circ}$ trans-saccadic test; $0.6^{\circ}-1.2^{\circ}$ for $0.6^{\circ}$ trans-saccadic test; symmetric about the test stimulus for the inter-saccadic tests). As most transsaccadic changes were not detected leading to a small dataset, data were pooled across observers before estimating the psychometric functions.

\section{Results and discussion}

After displaying the test and the reference stimulus, the subjects were asked three questions: (1) "Which displacement was larger?" (2) "Was there a change in interval 1?" and (3) "Was there a change in interval 2?". The latter two questions relate to detection performance that can be compared with Experiment 1. Detection data followed the general pattern of Experiment 1. Failure to trigger the saccade-contingent change resulted in catch trials that were used to assess the false alarm rate. As in Experiment 1, subjects acted conservatively and no false alarms were observed. Hit rates for trans-saccadic test stimuli were 0.14 (range 0.08-0.33) and 0.21 (range 0.13-0.33) for $0.6^{\circ}$ and $1.2^{\circ}$ displacements, respectively. Hit rates for inter-saccadic test stimuli were 0.88 (range 0.82-1.0) and 0.83 (range
$0.75-1.0)$ for $0.6^{\circ}$ and $1.2^{\circ}$ displacements, respectively. Hit rates were significantly lower (Newcombe 1998) for transsaccadic test stimuli than inter-saccadic test stimuli for both the $0.6^{\circ}$ and $1.2^{\circ}$ translation amplitudes $\left(\chi^{2}(1, N=139)=\right.$ $183.25, P<0.001$ and $\chi^{2}(1, N=129)=100.93, P<0.001$, respectively).

The hypothesis was that the apparent displacement of a detected trans-saccadic displacement would be judged as smaller than an equivalent sized inter-saccadic comparison stimulus. To evaluate this hypothesis, we needed to ensure that both stimuli were detected before evaluating the forced-choice data. Only trials where both the test and comparison stimulus transitions were triggered and detected were considered in the analysis of the psychometric functions for estimation of the point of subjective equality.

Figure 4 shows the psychometric functions for each of the $0.6^{\circ}$ test conditions. For the inter-saccadic test stimuli, it is clear that when the inter-saccadic comparison stimulus is larger than the test, it is more likely to be judged as larger and conversely when it is objectively smaller than the test then it is more likely to be judged as smaller. The point where psychometric function crosses chance probability of 0.5 is the point of subjective equality since the comparison stimulus cannot be distinguished from the test. Subjects equated inter-saccadic translations with similar size comparison stimuli as expected. However, point of subject equality for trans-saccadic test stimuli was shifted to lower translation sizes. In other words, trans-saccadic changes were judged as smaller than equivalent sized inter-saccadic changes. Inter-saccadic changes that were significantly smaller than the trans-saccadic were required for a percept of equality.

Thus, we conclude that detected trans-saccadic displacements appear smaller than equivalent inter-saccadic changes. As displacement is the stimulus for apparent motion, we propose that this trans-saccadic suppression of displacement underlies the reports of perceptual slowing in the first experiment. However, the point of subjective equality estimated in forced-choice procedures can be misleading. In a 2IFC comparison between a test stimulus and a reference, the $50 \%$ point is typically considered the point of subjective equality. The logic is that if a test stimulus is subjectively equivalent to the reference, the unbiased subject should respond by guessing which stimulus is larger (at the 50\% level). However, a 50\% point on the psychometric function could also arise from a bimodal (or multimodal) percept. For example, if the stimulus was typically seen as very small but occasionally as very large, then a reference between the two modes (but towards the smaller one) would be seen as larger on 50\% of trials, despite not being representative of either of the two actual percepts. Experiment 3 was designed to confirm the perceptual suppression 
(a) Probability of Seeing Test Stimulus as larger than Inter-Saccadic Comparison

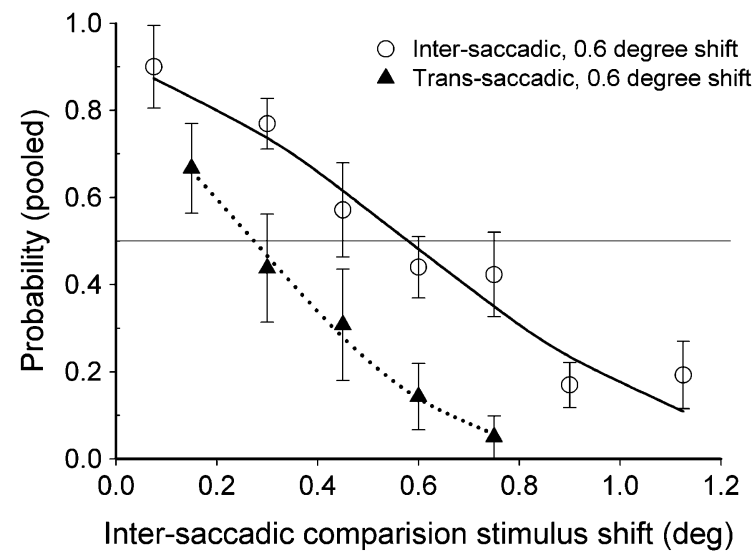

(b)

Proportion test trials reported larger than equivalent sized comparison (both stimuli reported seen)

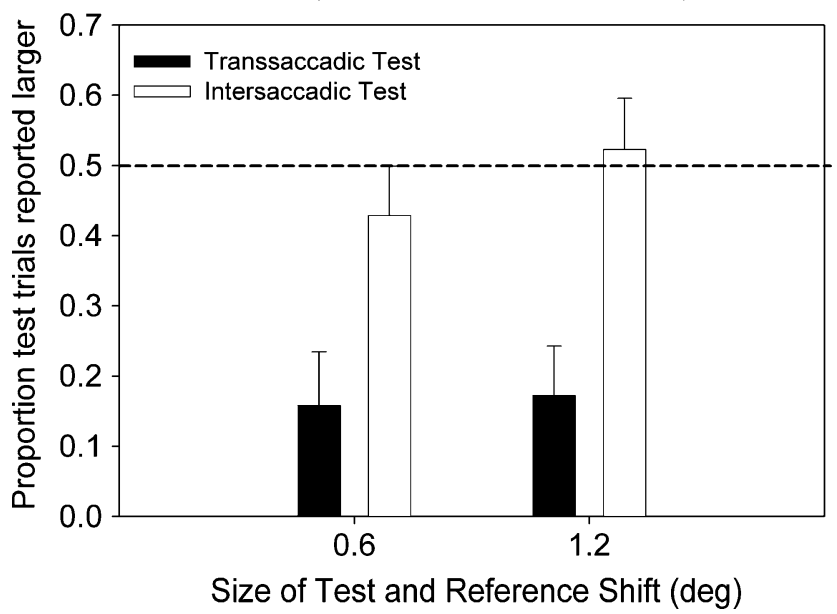

Fig. 4 Probability of seeing a test stimulus as larger than an intersaccadic comparison stimulus. Participants compared a 'test stimulus' in one interval against a 'comparison stimulus' in the other interval. Comparison stimuli were always inter-saccadic. Detected trans-saccadic changes (apparent motion) appeared smaller than equivalent intersaccadic changes based on the estimated point of subjective equality (PSE, intersection of the psychometric function with the horizontal line at 0.5 ). a The probability of seeing the test stimulus as larger than the inter-saccadic comparison is plotted against comparison size for the 0.60 test displacement trials. b Proportion of trials (with standard error) where the test stimulus was reported as larger than an equivalent sized comparison stimulus for the 0.60 and 1.20 displacement conditions

of trans-saccadic change magnitude with a different psychophysical method.

\section{Experiment 3: estimation of the magnitude of trans-saccadic changes}

There is a need to confirm that trans-saccadic changes are actually perceived as smaller than inter-saccadic changes using an alternative technique that provides a measure of apparent size of the translations. In this experiment, we used magnitude estimation to measure perceived translation magnitude on every trial and analysed the distribution of apparent displacements. If subjects truly see a slower or smaller change, then the distribution of perceived translation magnitudes should shift to lower values. As the perceived magnitude of the trans-saccadic image displacement may depend on its size in retinocentric coordinates (rather than purely allocentric or headcentric coordinates), we analysed separately conditions where the horizontal saccade was in the same direction as the shift (trans-saccadic retinal image displacement smaller than the saccade) and in the opposite direction (retinal image shift larger than the saccade). We also included vertical image shifts that were orthogonal to the horizontal component so that the horizontal retinal shift component was only determined by saccade size.

\section{Methods}

As in the previous experiments, subjects actively scanned natural images. Each trial started with a 1-s blank screen, and then the image was displayed for $4 \mathrm{~s}$. At some point during the trial, the image could be displaced either horizontally or vertically or not at all (catch trials). After subjects indicated their magnitude response (see below) the next trial began with another blank interval.

Displacements were in one of the four cardinal directions on the display (left, right, up and down) and one of three sizes $\left(0.4^{\circ}, 0.8^{\circ}\right.$ and $\left.1.2^{\circ}\right)$. On the one half of the noncatch trials, transitions were trans-saccadic and on the other half, transitions were inter-saccadic, taking place $150 \mathrm{~ms}$ after a saccade was detected and before another took place (see "General methods"). Trials were blocked by transition type (horizontal vs. vertical translation) and presented in counterbalanced order. Each block lasted about $10 \mathrm{~min}$. Within each block, the trials were randomized with respect to size and direction of transition. Subjects were shown 160 trials (12 repeats of each condition plus 16 catch trials) across two sessions each consisting of a horizontal block of 40 trials and a vertical block of 40 trials.

On each trial, subjects made judgments of the apparent size of the transitions on an increasing scale from 0 to 9 using a method of magnitude estimation (Stevens 1956). A $0.8^{\circ}$ inter-saccadic translation was assigned a value of 5 on the scale as a "standard" or reference. Subjects scaled their estimates of perceived displacement magnitude relative to the reference and were further told to respond with a ' 0 ' if no change was detected. Two examples of the horizontal standard (always $0.8^{\circ}$ inter-saccadic to the right) were shown at the beginning of the horizontal blocks, and two examples of the vertical standard (always 
$0.8^{\circ}$ inter-saccadic upward) were shown at the beginning of the vertical blocks.

Results and discussion

In total, there were 1,600 trials collected across the ten subjects. Trials contaminated by blinks, tracking errors, and failures to trigger (or triggering on saccades that did not meet the criteria) were eliminated manually from the data during a data-screening step (resulting in pruning of 534 trials). There was no systematic relation between these eliminated trials and particular images or displacement conditions. The average amplitude of saccades that triggered a transition was $24.5^{\circ}$ and positively skewed due to the velocity threshold (median $18.9^{\circ}$, lower quartile $11.5^{\circ}$, upper quartile $29.4^{\circ}$ ). The transitions were triggered on the horizontal component but there were also vertical components on many saccades (median of vertical component $-0.7^{\circ}$, lower quartile $-4.5^{\circ}$, upper quartile $3.1^{\circ}$ ).

\section{Detection rate}

Data were collapsed across both sessions, as there was no significant difference in performance between sessions $(P>0.15)$. On average, $87.6 \pm 1.08 \%$ (mean $\pm 95 \% \mathrm{CI})$ of the triggered inter-saccadic changes were detected, and $40.9 \pm 4.32 \%$ of trans-saccadic ones were detected across all subjects and conditions. Unlike Experiments 1 and 2, the false alarm rate was not zero and subjects erroneously reported detecting a translation on $4.6 \%$ of the catch trials.

In both inter-saccadic and trans-saccadic trials, the proportion of detected saccades increased with amplitude of the transition (see Table 1 for a summary of detection rates). Although trans-saccadic detection rates were slightly higher for vertical than for horizontal stimuli at each transitions size, there were no statistically significant differences in detection rate for trans-saccadic vertical shifts compared to trans-saccadic horizontal shifts $\left[\chi^{2}(1)=1.3945\right.$, $P=0.2377]$.

McConkie and Currie (1996) reported that observers were more sensitive to trans-saccadic transitions in the same direction as the saccade compared to the opposite direction, while Bridgeman and Macknik (1995) found the reverse effect. Contrary to these earlier reports, there was no significant difference between the detectability of trans-saccadic horizontal changes in the direction of the horizontal component of the saccade and those opposite the saccade $\left[\chi^{2}(1)=1.0239, P=0.3116\right]$. In the inter-saccadic condition, subjects were much more likely to detect the stimulus transitions compared to the trans-saccadic conditions and the difference between inter-saccadic and trans-saccadic detection rates was significant for all transitions sizes and both horizontal and vertical directions. The results are consistent with Experiment 1 and indicate significant suppression of trans-saccadic displacement of natural images.

\section{Magnitude estimates}

Based on Experiment 2, the hypothesis was that subjects would perceive detectable transitions as smaller during trans-saccadic intervals compared to inter-saccadic intervals. Thus, analysis of the magnitude estimation data was restricted to the detected cases.

From the detected cases, the mean magnitude estimate for horizontal and vertical scene transitions was calculated and compared (Fig. 5). Repeated measures ANOVAs indicated a significant main effect of session for horizontal $[F(1,8)=4.89, P=0.058]$ and vertical estimates $[F(1,8)=$ $8.29, P=0.021)]$. This effect was due to estimates being, on average, slightly larger in the second session (approximately one half unit on the magnitude scale). However, there were no significant interactions between session and any other variable. It was also observed that the average magnitude estimates were smaller for horizontal than vertical shifts. The mean magnitude estimate for horizontal
Table 1 Detection rates for the conditions in Experiment 3 as a function of saccade contingency and transition size

The final column shows the results of a 2-sample test for equality of proportions with continuity correction between trans-saccadic and intersaccadic conditions

\begin{tabular}{llll}
\hline Transition size & $\begin{array}{l}\text { Trans-saccadic } \\
\text { detection } \\
(\text { mean } \pm 0.95 \mathrm{CI})\end{array}$ & $\begin{array}{l}\text { Inter-saccadic } \\
\text { detection } \\
(\text { mean } \pm 0.95 \mathrm{CI})\end{array}$ & $\begin{array}{l}\text { Inter-saccadic versus } \\
\text { trans-saccadic difference }\end{array}$ \\
\hline Horizontal (deg) & & & \\
0.4 & $26.9 \pm 4.32$ & $81.0 \pm 2.85$ & $\chi^{2}(1)=51.92, P<0.0001$ \\
0.8 & $45.2 \pm 5.07$ & $90.3 \pm 1.78$ & $\chi^{2}(1)=42.79, P<0.0001$ \\
1.2 & $47.5 \pm 5.13$ & $92.8 \pm 1.74$ & $\chi^{2}(1)=35.57, P<0.0001$ \\
Vertical (deg) & & & \\
0.4 & $35.3 \pm 3.01$ & $78.73 \pm 2.82$ & $\chi^{2}(1)=34.53, P<0.0001$ \\
0.8 & $45.6 \pm 6.13$ & $89.0 \pm 2.02$ & $\chi^{2}(1)=33.86, P<0.0001$ \\
1.2 & $49.9 \pm 5.39$ & $96.2 \pm 1.31$ & $\chi^{2}(1)=43.93, P<0.0001$ \\
\hline
\end{tabular}




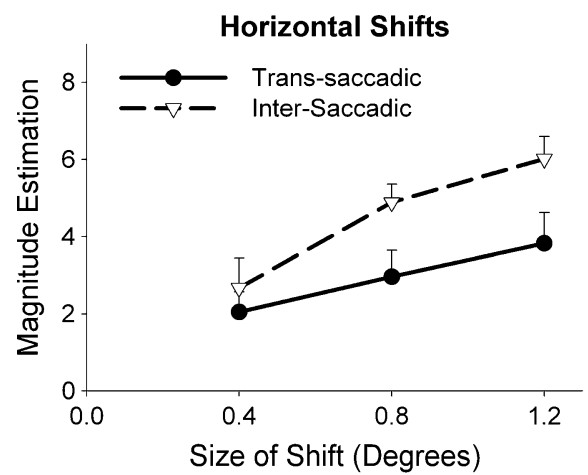

Fig. 5 Mean magnitude estimates across all subjects. The abscissa is actual size of change in degrees, and the ordinate is the magnitude estimation from 0 to 9. Left-hand panel shows mean magnitude estimates for horizontal shifts. Right-hand panel shows mean magnitude

shifts were 3.38 (trans-saccadic) and 4.56 (inter-saccadic) compared to mean magnitude estimate for vertical shifts of 4.28 (trans-saccadic) and 4.91 (inter-saccadic).

We were primarily interested in the effects of saccade contingency of the transition. The ANOVA indicated a significant interaction between the explanatory variables of transition magnitude and saccade contingency for both horizontal $[F(1,9)=9.28, P=0.0139]$ and vertical magnitude estimates $[F(1,9)=27.22, P=0.0005]$. The main effects marginal to this interaction were also significant for both the horizontal transitions $[F(1,6)=71.33, P<0.0001$ and $F(1,6)=60.83, P=0.0002$ for transition size and contingency, respectively $]$ and the vertical transitions $[F(1,7)=$ 73.73, $P<0.0001$ and $F(1,6)=20.52, P=0.0040$ for transition size and contingency, respectively]. The nature of the interaction can be observed in Fig. 5. For both horizontal and vertical transitions the simple main effects of transition size are seen with the estimates increasing with transition size for all contingency by transition-direction conditions and the interaction was apparent in the divergence of the plots for inter-saccadic and trans-saccadic stimuli at larger transition sizes. For the horizontal transitions, magnitude estimates increased with the transition size but more steeply for inter-saccadic than trans-saccadic transitions. Post hoc comparisons (multiple comparisons controlled for the family-wise error rate) indicated that the inter-saccadic estimates were on average larger than the trans-saccadic estimates by $0.35 \pm 0.926$ (mean \pm 0.95 CI), $1.74 \pm 0.789$ and $1.91 \pm 0.808$ for the $0.4^{\circ}, 0.8^{\circ}$ and $1.2^{\circ}$ shifts, respectively, although the difference was only significant for the larger two transitions $(P=0.754$, $P<0.0001, P<0.001$, respectively). Similar analysis for the vertical transitions indicated a significant difference only at the largest transition size with mean inter-saccadic estimates larger than trans-saccadic estimates by $0.58 \pm 0.832,0.44 \pm 0.775$ and $1.65 \pm 0.784$ for the $0.4^{\circ}$,
Vertical Shifts

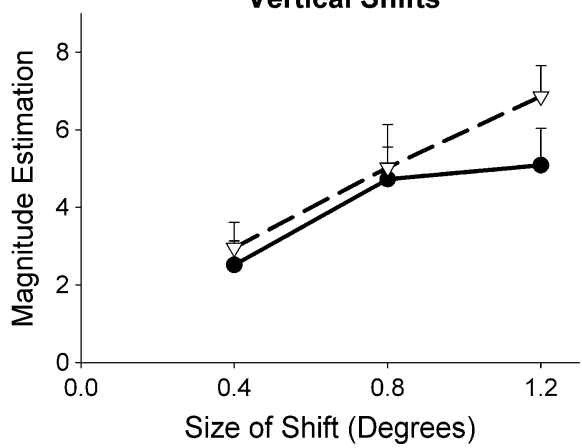

estimates for vertical shifts. All changes were triggered on horizontal saccades. Inter-saccadic shifts are shown as open symbols and dashed lines and trans-saccadic shifts as solid symbols and lines. Error bars indicate $95 \%$ confidence intervals

$0.8^{\circ}$ and $1.2^{\circ}$ shifts, respectively $(P=0.255, P=0.447$ and $P<0.0001$ respectively). ${ }^{2}$

Although previous studies have found differences in detection rate for trans-saccadic displacements in the same direction as the saccade versus oppositely directed displacements, as noted in the previous section we found no such differences. Consistent with this detection finding, there was also no difference in magnitude estimates for translations directed along as opposed to against the saccade for horizontal shifts $[F(1,8)=2.3034, P=0.1676]$ or for different direction of vertical shifts $[F(1,8)=0.0020$, $P=0.9657]$ and there were also no significant interactions between direction and size of transition or saccade. Further, there was no significant effect of saccade amplitude (or the horizontal or vertical components of the saccade) on the magnitude estimates. The latter finding is likely due to the limited range of saccade amplitudes resulting from triggering on only large saccades as Experiment 1 found that increasing saccade duration (and amplitude) reduced transsaccadic detection rates.

If the apparent displacement of detected trans-saccadic shifts was smaller than objectively equivalent inter-saccadic changes, then the magnitude estimates for the former transitions should be lower than for the latter. This was confirmed for both horizontal and vertical transitions. Thus, subjects were not only more likely to detect inter-saccadic transitions than trans-saccadic transitions but also reported

\footnotetext{
${ }^{2}$ These findings were confirmed with non-parametric statistical methods because some of the histograms were not normally distributed. The effect of transition size and saccade contingency were significant for horizontal [Kruskal-Wallis $\chi^{2}(1)=21.6896, P<0.0001$ ] and vertical conditions (Kruskal-Wallis $\chi^{2}(1)=5.0243, P=0.025$ ). Post hoc nonparametric comparisons (corrected for multiple comparisons) confirmed that inter-saccadic estimates were significantly larger than trans-saccadic estimates for $1.2^{\circ}$ and $0.8^{\circ}$ but not $0.4^{\circ}$ horizontal transitions and for $1.2^{\circ}$ but not for $0.4^{\circ}$ and $0.8^{\circ}$ vertical transitions.
} 
larger magnitude estimates for inter-saccadic than transsaccadic conditions when they detected the changes. However, there was a strong interaction and these differences were only significant at the larger transition sizes. Moreover, the difference between inter-saccadic and trans-saccadic estimates increased with transition size over the range tested. It is worth remembering that detection rates were lowest for the $0.4^{\circ}$ trans-saccadic transitions and the difficulty in simply detecting the stimulus and floor effects due to the scale could be a factor in the non-significant effects of contingency on magnitude estimates at the smallest transitions.

Histograms of pooled trans-saccadic magnitude estimates for detected trials were analysed for indications of bimodality. Hartigan's dip test provided no indication of significant deviations $(P>0.05)$ from unimodality for all conditions (Hartigan and Hartigan 1985). In general, the peaks of the histograms were shifted towards smaller magnitude estimates during saccades compared to during fixation.

The magnitude estimation results are consistent with those of Experiment 2 and indicate a perceived reduction in the size of an apparent motion transition during saccades. This confirms anecdotal reports as well as experiments with simple stimuli (Festinger and Holtzman 1978; Ilg and Hoffmann 1993) and suggests that during a saccade the perceived magnitude of the trans-saccadic change is reduced as well as its visibility. This can be considered a generalized form of trans-saccadic suppression of displacement (SSD). This SSD was more pronounced for horizontal transitions than for vertical transitions that occurred during horizontal saccades consistent with earlier reports (Heywood and Churcher 1981). Previous research has suggested that changes orthogonal to the saccade direction are easier to detect than congruent changes (Festinger and Holtzman 1978; Niemeier et al. 2003) although others have reported no such difference (Mack 1970; Stark et al. 1976; Bridgeman and Stark 1979). Our results suggest that perceived magnitude of trans-saccadic displacements may exhibit a similar congruent versus orthogonal anisotropy. Further investigation with vertical as well as horizontal saccades would be required to confirm this hypothesis.

\section{General discussion}

In summary, we found that trans-saccadic changes are suppressed for stimuli filling a large part of the visual field, particularly for long duration saccades. The suppression results in reduced detectability and phenomenological slowing of detected transitions. We conclude that the phenomenological slowing of the apparent motion during saccades is consistent with our findings of reduced apparent magnitude of trans-saccadic displacements compared to displacements during fixation. Most previous studies of trans-saccadic displacement or trans-saccadic motion perception have used small stimuli. For instance, Bridgeman et al. (1975) studied the displacement of a $13 \times 13^{\circ}$ field of dots and others have used dot, grating or line stimuli (Campbell and Wurtz 1978; Brooks et al. 1980; Burr et al. 1982; Ilg and Hoffmann 1993; Castet and Masson 2000). In this study, we studied saccadic sensitivity to displacement of a large-field display. Considered as an apparent motion stimulus, it simulates the expected motion in the visual field during a saccadic eye movement or a head rotation. When the subject moves bodily the entire visual field is transformed. Since the eye is approximately spherical, rotation of the eye will result in an angular displacement of the entire retinal image. We confirmed that observers are not sensitive to such displacement when it occurs during a saccadic eye movement. This confirms earlier findings of saccadic suppression of displacement with smaller, simpler stimuli $^{3}$ (Bridgeman et al. 1975; Li and Matin 1997; MacAskill et al. 2000, 2003; Anand and Bridgeman 2002; Deubel et al. 2004). Bridgeman and Fisher (1990) reported that sensitivity to trans-saccadic target displacement depended on target eccentricity with more suppression for central than peripheral targets. Perisaccadic compression of space is also reportedly centred and most pronounced near the saccade landing point (Awater et al. 2005). In our stimulus the target extended across much of the visual field and the suppression of displacement observed suggests that saccadic suppression also occurs during viewing of extended natural images. Thus, it is likely that saccadic suppression of displacement is active during natural viewing of complex scenes.

In their classic study of trans-saccadic motion sensitivity, Burr et al. (1982) noted that even supra-threshold motion stimuli appeared muted trans-saccadically. Ilg and Hoffmann (1993) had similar findings and noted that their subjects were responding to the relative motion of the saccade target compared to the background. MacAskill et al. (2003) argued that degree of saccadic suppression of displacement is larger when subjects judge the whole motion of a display compared to the relative motion of a saccade target to a background. Our experiments confirm the reduced salience and phenomenological slowing of transsaccadic translations. Further, we extend these findings by: considering the whole-field apparent motions of natural scenes, studying absolute motion of the visual world rather than relative motion, and measuring the degree of apparent

\footnotetext{
${ }^{3}$ Previous studies used a wide range of stimuli from small dots to moderate sized (e.g. $13^{\circ} \times 13^{\circ}$ in Bridgeman et al. 1975). Ilg and Hoffmann (1993) used a simple dot pattern but a large display.
} 
slowing reported anecdotally by Ilg and Hoffman and Burr et al.

As suggested by earlier researchers (e.g. Li and Matin 1997), it is informative to distinguish between saccadic suppression of displacement, saccadic suppression of visibility and saccadic suppression of motion. During intersaccadic transitions, the displacement of the image is an apparent motion stimulus and displacement of the image is associated with a strong sense of apparent motion at least for modest translations. During trans-saccadic displacements, it is possible for a dissociation between the apparent motion and apparent displacement for a given image translation. During saccades the visual world streams across the retina at several hundred degrees per second. One might suppose that saccadic suppression of the apparent motion during saccades reflects general suppression of motion signals during saccades. However, the large retinal image motion during the saccade results in considerable motion blur, obscuring and reducing contrast of fine detail (Campbell and Wurtz 1978). This motion blur could obscure the more subtle apparent motion associated with the image displacement stimulus. If the apparent motion signal or even visibility of the image were essentially below threshold during the saccade due to blur, masking or active suppression then estimation of trans-saccadic displacement would necessarily depend on comparison of image position before and after the saccade (MacAskill et al. 2003). The loss of this motion signal and the requirement to carry out these comparisons in other frames of reference than retinocentric coordinates (see below) likely makes the image translation easier for the visual system to discount during saccades.

The image translation to be detected is a shift of the entire scene in head-centric coordinates. During fixation, detection of the stimulus apparent motion is equivalent to detection of motion or positional shift on the retina. Shioiri and Cavanagh (1989) found that the detectability of twoframe apparent motion of a random-dot field was greatly reduced during a saccade, regardless of whether the motion was specified in retinal or spatial coordinates and even though apparent motion could be seen for ISI longer than the saccade duration (Braddick 1973; Baker and Braddick 1985). However, to estimate displacement magnitude during saccadic eye movements, the motion detection or comparison of stimulus position must account for the motion of the eye, even if the stimulus is visible. Thus, these comparisons must be performed in other frames of reference than retinocentric coordinates (i.e. image displacement must be judged in head-centric-or perhaps allocentric coordinates if the head moves in a natural setting-as even stationary features change position on the retina during the eye movement). If the eye is moving and the stimulus visible this would increase noise since the subject would need to detect a small change imposed on a large velocity signal and to account for the expected velocity profile of the eye movement. If the trans-saccadic stimulus is actively suppressed, reduced in visibility due to motion blur, or masked by more salient pre- and post-saccadic images, then the subject would need to compare the difference in position prior to and following the saccade. Thus, the visual system would need to account for the size of the saccade and thus the expected retinal displacement of a stationary scene. This is a difficult process since it relies on 'knowledge' of the saccade size executed, and is sensitive to motor error, accuracy of efferent/afferent eye position estimates, and precision and maintenance of memory of the previous position (alternatively the previous position could be 'updated' to the expected retinal position in the new gaze position). Thus, we may expect that the trans-saccadic signal is weaker, noisier and less precise and this may contribute to the lower detection rate of trans-saccadic apparent motion and to its apparent slowing relative to apparent motion during fixation.

Conversely, one possible role for saccadic suppression of displacement in such natural scenes is to accommodate mismatch between retinal image displacement and expected retinal image displacement associated with the executed eye movement. Theories of spatial updating and visual stability across fixations have often postulated a role for CD or efference copy (Sperry 1950; Von Holst and Mittelstaedt 1950; Wurtz 2008). In one version of this account, visual stability is accomplished via a CD signal that updates spatial maps. This updating-or any other conceivable extra-retinal mechanism to account for the motion generated by the eye movement-is subject to noise and error. Thus, saccadic suppression of displacement could serve to suppress spurious residual displacement and motion signals. Bridgeman et al. (1975) posited such a suggestion to account for their findings of saccadic suppression of displacement: "Our data can be reconciled with $\mathrm{CD}$ theories if it is assumed that the error between the extent of a saccade and the corresponding retinal image displacement must reach a threshold before a displacement of the world is detected. With this assumption, the computed comparison need to be only fast and accurate enough to maintain visual-motor coordination, but the existence of such a computation would still account for the phenomena classically cited in support of CD theories. Saccadic suppression could inhibit perception when the $\mathrm{CD}$ and visual information do not match."

What putative mechanism of saccadic suppression best explains the current results? The subjects were given a task that was essentially head (or allo) centric, as they judged the apparent motion of the image in the world. However, they could have performed the task based on the transient, retino-centric apparent motion signal that was available to the observer during the inter-saccadic stimuli but subject to 
saccadic suppression during the trans-saccadic stimuli. Active suppression of the transient inter-saccadic motion signal during the saccade would make it less detectable. It is possible but less clear that it could reduce the magnitude of the apparent motion percept due to attenuation of the signal. If the subject was using this motion signal, then we would expect it to be affected by the parameters of the saccade (Macknik et al. 1991). Given the lack of any effect of eye movement parameters such as saccade direction (relative to the shift) and vertical component suggests that the observers were judging the displacement or possibly apparent motion of the image across the saccade rather than the transient motion signal. Similar arguments would argue against a role of trans-saccadic motion blur and reduced illumination.

Given the rich, natural images used in the present experiments, post-saccadic masking is more likely to be a factor than active suppression. Masking mechanisms would also reduce the visibility of the apparent motion signal and hence detectability. These mechanisms also introduce a temporal 'gap' between post- and pre-saccadic stimuli that could weaken the apparent motion signal. Thus, effects on detection and perhaps magnitude estimation are consistent with masking. McConkie and Currie (1996) proposed that detection of displacement in natural scenes is based on local information at the landing point of the saccade. They argue that this type of model combined with tendency for saccadic undershoot predicts better detection performance when the displacement is in the direction of the saccade compared to oppositely directed. No such difference was found in the current study.

Masking or active saccadic suppression could factor in the present experiments by eliminating the ability to perform the task in a retino-centric frame of reference through suppression of the transient motion signal. Performing the task in head-centric coordinates requires taking account the eye movement. Mismatch between efferent copy and actual motion could be attributed to stimulus motion but also to eye movements that differed from those planned. Thus, perceived reduction of trans-saccadic displacement magnitudes is consistent with attribution of some of the image shift to the eye movement itself. Similarly, signal to noise considerations predict the effects of transition size (relative to the saccade) on detection. Finally, to accomplish the head-centric task, the displacement of the target is judged relative to the initial position taking into account the eye movement. There is no reason to presume that whether the eye moves with or against the displacement has any effect on the difficulty of this judgement. On the other hand, for purely orthogonal shifts, there is no saccade imposed motion in the direction of the shift to be accounted for, which could explain the reduced effects of saccades on magnitude estimates for orthogonal shifts compared to shifts along the saccade direction. Note that this conclusion must be tempered by the fact that orthogonality was inherently confounded with direction in Experiment 3.

Thus, on the whole, our findings are most consistent with subjects making allocentric judgements. As such, transsaccadic effects on detection and displacement magnitude reflected uncertainty in efference copy, spatial updating or cancellation. Subjects were forced to make trans-saccadic judgements of displacement rather than retinal motion due to masking and possibly active suppression of the transsaccadic stimulus.

In terms of practical application of this work, scene changes in realistic images presented in virtual environments can be suppressed during saccadic eye movements. Our system was similar to a normal virtual-environment display without any special hardware except for the video-based eye tracker. We have previously argued that saccadic suppression can be used to perceptually hide potentially disruptive graphics updates in virtual environments-or conversely to avoid doing so when monitoring a display for changes in state (Schumacher et al. 2004). To hide updates during a saccade, there must be sufficient time after saccade detection to complete the change before the end of the eye movement. In the current implementation, this limits us in triggering on fairly large saccades, which impacts the maximum update rate. Ideally, sampling rate would be higher than the $120 \mathrm{~Hz}$ used here as the limited sampling rate means that the estimate of the size of the saccade and prediction of whether a graphic update will be perceptible must be based upon only a few samples from the beginning of a saccade. Detected transsaccadic changes (motion) appear slower than equivalent inter-saccadic changes. Thus, even if updates are not completely suppressed they are much less salient.

Acknowledgments The support of the Natural Science and Engineering Research Council and the Ontario Centres of Excellence is greatly appreciated. Thanks to Margarita Vinnikov for assistance with the programming and to Judy Sam and Linda Tomkins for assistance with the analysis.

\section{Appendix: Saccade detection and prediction}

Most previous studies of saccade-contingent updates have been performed using simple displays or desktop monitors (McConkie and Currie 1996; MacAskill et al. 2003) with simple objects (Deubel et al. 1998) as saccade targets. It is not clear how well these results generalize to complex real world scenes or a virtual environment. It is also unclear whether a video-based eye-tracking system with a temporal resolution of $120 \mathrm{~Hz}$ is able to detect saccades fast enough to perform scene changes in a realistic environment. To investigate these issues, we studied gaze behaviour while viewing complex scenes. 
Subjects

Nine normal subjects participated, aged from 21 to 34 years. All were naïve with respect to the task and inexperienced with the equipment. Three of them performed the experiment twice in order to evaluate whether the findings were repeatable across two different sessions for the same subject.

\section{Method}

In each session, five arbitrarily chosen scenes were sequentially shown to the subjects for $30 \mathrm{~s}$ each while their eye movements were recorded. Subjects began the session fixating a white dot target centred in the middle of the otherwise black display. This fixation stimulus was displayed for $2.0 \mathrm{~s}$ before and after each trial. Eye movement data were monitored during these fixations to ensure that the system remained correctly calibrated.

The distance from the eyes to the screen was $80 \mathrm{~cm}$ and viewing was binocular. Subjects were told to analyse and memorize the scenes for a later memory task. Even though no scene changes were imposed, we asked them to watch out for possible scene changes and press a button as soon as they detected one. We did this to avoid possible difference in eye movement behaviour when this additional task was required in the main experiment. While the subjects moved their gaze over the picture, the eye position data were recorded.

\section{Results}

The recorded eye movement data consisted of the eye position and a flag indicating the occurrence of an eye blink for each sample instant. The current velocity was estimated using two differentiators, a first-order backward difference and a smoothed five-point differentiator.

The collected data were evaluated to determine the frequency of saccades made during free viewing of scenes in a virtual environment and to develop and optimize our detection algorithm. Based on their duration, the measured saccades were classified into three categories-short, medium, long-with durations of less than $42 \mathrm{~ms}, 42-58 \mathrm{~ms}$, and greater than $58 \mathrm{~ms}$, respectively. While viewing the five scenes (total time $150 \mathrm{~s}$ ), on average subjects made $249.5 \pm 52.4$ saccades (mean \pm standard deviation). The distribution of saccades in the different classes was consistent across subjects with $79.08 \pm 28.10$ small, $85.67 \pm 17.70$ medium, and $84.75 \pm 24.30$ long duration saccades. Since we are primarily interested in the class of long-duration saccades, this would indicate that scenes could be changed about 0.56 times/s or every $1.7 \mathrm{~s}$ assuming that all saccades are detectable.
Only saccades in the long-duration category, with duration longer than $58 \mathrm{~ms}$, were sufficiently long to be used for saccade-contingent changes in our experimental setup. Due to system latency, a saccade-contingent change needed to be initiated by the third eye tracker sample (frame) from the start of such a large saccade. In order to determine a reasonable threshold value that has to be exceeded to trigger a change, we evaluated the velocity at this period in time for long-duration saccades. The results indicated that a peak velocity value of greater $200 \%$ s corresponded to saccades that were appropriately long. These values are consistent with values determined from earlier studies of saccadic profiles (Leigh and Zee 1999). Our results are generally consistent with scan path behaviour in other studies that used complex images (Yarbus 1967).

\section{Detection algorithm evaluation}

The eye position was sampled with a frequency of $120 \mathrm{~Hz}$; successive samples were $8.33 \mathrm{~ms}$ apart. The algorithm used was based upon a five-point differentiator that worked on a window of estimates from five successive frames and calculates the filtered velocity for the value in the middle. This filter is symmetric about its center and thus adds a delay of 2.5 frames. A saccade was indicated if the filtered velocity exceeded a threshold of $60 \%$ s. The velocity threshold was based on typical saccadic profiles and the peak velocity distribution for 'long' duration saccades (see previous section). However, if the velocity exceeded $900^{\circ}$ s at the first or second sample point, the saccade detector was not triggered as such a rapid acceleration is physiologically unlikely. Screening and removal of these latter cases and sanity checks with another velocity threshold based on a simple two-point differentiator made the system more robust against noise and other artefacts (e.g. undetected eye-blinks). In order to guarantee that an update was not triggered at the saccade peak velocity, the acceleration was estimated over the previous three frames of data in order to verify that it was continually increasing. A change was only triggered if the velocity criterion and the acceleration criterion were simultaneously met.

A saccade duration of $58 \mathrm{~ms}$ was set as a lower bound limit for making a trans-saccadic change. The false alarm rate (triggering for saccades less than $58 \mathrm{~ms}$ in duration or for eye tracker artefacts) for the algorithm was $16.2 \%$. A low false alarm rate should be the highest priority. Even though the algorithm is fairly conservative, the miss rate remained low enough to allow a reasonable frequency of saccade-contingent change opportunities. Data from all detected trials were screened visually to identify and remove false alarms. 


\section{References}

Anand S, Bridgeman B (2002) An unbiased measure of the contributions of chroma and luminance to saccadic suppression of displacement. Exp Brain Res 142:335-341

Awater H, Burr D, Lappe M, Morrone MC, Goldberg ME (2005) Effect of saccadic adaptation on localization of visual targets. J Neurophysiol 93:3605-3614

Baker CL Jr, Braddick OJ (1985) Temporal properties of the shortrange process in apparent motion. Perception 14:181-192

Braddick O (1973) The masking of apparent motion in random-dot patterns. Vision Res 13:355-369

Bridgeman B, Fisher B (1990) Saccadic suppression of displacement is strongest in central vision. Perception 19:103-111

Bridgeman B, Macknik SL (1995) Saccadic suppression relies on luminance information. Psychol Res 58:163-168

Bridgeman B, Stark L (1979) Omnidirectional increase in threshold for image shifts during saccadic eye movements. Percept Psychophys 25:241-243

Bridgeman B, Hendry D, Stark L (1975) Failure to detect displacement of the visual world during saccadic eye movements. Vision Res 15:719-722

Brooks BA, Yates JT, Coleman RD (1980) Perception of images moving at saccadic velocities during saccades and during fixation. Exp Brain Res 40:71-78

Burr DC, Holt J, Johnstone JR, Ross J (1982) Selective depression of motion sensitivity during saccades. J Physiol 333:1-15

Burr DC, Morrone MC, Ross J (1994) Selective suppression of the magnocellular visual pathway during saccadic eye movements. Nature 371:511-513

Burr DC, Morgan MJ, Morrone MC (1999) Saccadic suppression precedes visual motion analysis. Curr Biol 9:1207-1209

Campbell F, Wurtz RH (1978) Saccadic omission: why we do not see a grey-out during a saccadic eye movement. Vis Res 18:1297-1303

Castet E, Masson GS (2000) Motion perception during saccadic eye movements. Nat Neurosci 3:177-183

Deubel H, Bridgeman B, Schneider WX (1998) Immediate post-saccadic information mediates space constancy. Vision Res 38:3147-3159

Deubel H, Bridgeman B, Schneider WX (2004) Different effects of eyelid blinks and target blanking on saccadic suppression of displacement. Percept Psychophys 66:772-778

Diamond MR, Ross J, Morrone MC (2000) Extraretinal control of saccadic suppression. J Neurosci 20:3449-3455

Dodge R (1903) Five types of eye movements in the horizontal meridian plane of the field of regard. Am J Physiol 8:307-329

Dodge R (1905) The Illusion of clear vision during eye movement. Psychol Bull 3:193-199

Festinger L, Holtzman JD (1978) Retinal image smear as a source of information about magnitude of eye movement. J Exp Psychol Hum Percept Perform 4:573-585

Garcia-Perez MA (2001) Yes-no staircases with fixed step sizes: psychometric properties and optimal setup. Optom Vis Sci 78:56-64

Hartigan JA, Hartigan PM (1985) The dip test of unimodality. Ann Stat 13

Heywood S, Churcher J (1981) Direction-specific and position-specific effects upon detection of displacements during saccadic eye movements. Vis Res 21:255-261

Holt E (1903) Eye movements and central anesthesia. Harvard Psychol Stud 1:3-45

Ilg UJ, Hoffmann KP (1993) Motion perception during saccades. Vision Res 33:211-220

Irwin DE (1992) Memory for position and identity across eye movements. J Exp Psychol Learn Mem Cogn 18:307-317

Kleiser R, Seitz RJ, Krekelberg B (2004) Neural correlates of saccadic suppression in humans. Curr Biol 14:386-390
Leigh RJ, Zee DS (1999) The neurology of eye movements, 3rd edn edn. Oxford University Press, New York

Li WX, Matin L (1990) The influence of saccade length on the saccadic suppression of displacement detection. Percept Psychophys 48:453-458

Li W, Matin L (1997) Saccadic suppression of displacement: separate influences of saccade size and of target retinal eccentricity. Vision Res 37:1779-1797

MacAskill MR, Anderson TJ, Jones RD (2000) Suppression of displacement in severely slowed saccades. Vision Res 40:3405-3413

MacAskill MR, Jones RD, Anderson TJ (2003) Saccadic suppression of displacement: effects of illumination and background manipulation. Perception 32:463-474

Mack A (1970) An investigation of the relationship between eye and retinal image movement in the perception of movement. Percept Psychophys 8:291-298

MacKay DM (1970) Elevation of visual threshold by displacement of retinal image. Nature 225

Macknik SL, Fisher BD, Bridgeman B (1991) Flicker distorts visual space constancy. Vision Res 31:2057-2064

Matin E (1974) Saccadic suppression: a review and an analysis. Psychol Bull 81:899-917

McConkie GW, Currie CB (1996) Visual stability across saccades while viewing complex pictures. J Exp Psychol Hum Percept Perform 22:563-581

Newcombe RG (1998) Interval estimation for the difference between independent proportions: comparison of eleven methods. Stat Med 17:873-890

Niemeier M, Crawford JD, Tweed DB (2003) Optimal transsaccadic integration explains distorted spatial perception. Nature 422:76-80

Ross J, Burr D, Morrone C (1996) Suppression of the magnocellular pathway during saccades. Behav Brain Res 80:1-8

Schumacher J, Allison RS, Herpers R (2004) Using saccadic suppression to hide graphic updates. In: Eurographic/ACM SIGGRAPH Symposium on Virtual Environments 2004. ACM SIGGRAPH, Grenoble, France, pp 17-24

Shioiri S, Cavanagh P (1989) Saccadic suppression of low-level motion. Vision Res 29:915-928

Sperry RW (1950) Neural basis of the spontaneous optokinetic response produced by visual inversion. J Comp Physiol Psychol 43:482-489

Stark L, Kong R, Schwartz S, Hendry D (1976) Saccadic suppression of image displacement. Vision Res 16:1185-1187

Stevens SS (1956) The direct estimation of sensory magnitudes-loudness. Am J Psychol LXIX:1-25

Thiele A, Henning P, Kubischik M, Hoffmann KP (2002) Neural mechanisms of saccadic suppression. Science 295:2460-2462

Thilo KV, Santoro L, Walsh V, Blakemore C (2004) The site of saccadic suppression. Nat Neurosci 7:13-14

Triesch J, Ballard DH, Hayhoe MM, Sullivan BT (2003) What you see is what you need. J Vis 3:86-94

Uchikawa K, Sato M (1995) Saccadic suppression of achromatic and chromatic responses measured by increment-threshold spectral sensitivity. J Opt Soc Am A Opt Image Sci Vis 12:661-666

Volkmann FC, Riggs LA, White KD, Moore RK (1978) Contrast sensitivity during saccadic eye movements. Vision Res 18:1193-1199

Von Holst E, Mittelstaedt H (1950) Das Reafferenzprinzip. Naturwissenschaften 37:464-476

Wurtz RH (2008) Neuronal mechanisms of visual stability. Vision Res 40:2070-2089

Yarbus AL (1967) Eye movements and vision, translation by L. A. Riggs. Plenum, New York 